\title{
The Detection for Nickel Base Super Alloy Based on Ultrasonic Phased Array
}

\author{
Mengqiu Gao, Bing Li, Lei Chen, and Yuegen Wang
}

\begin{abstract}
The material of the gas turbine blade is nickel base super alloy. When the blades are inspected the artificial evaluation can only be adopted to normal inspection due to the lack of industry benchmarks, which contributes to single indicator and large error. To solve this troublesome problem and help inspect the blades, this paper proposes a method of data analysis and processing. At first, the defects were recognized from the detected image, and then quantitation, qualification and location analysis were made from the recognized defects. Through comparing with the artificial evaluation, this method is a good one which can provide more parameters, has high accuracy and much reliability.
\end{abstract}

Index Terms - Gas turbine, image processing, defect analysis, nickel base super alloy.

\section{INTRODUCTION}

Ultrasonic phased array technology originated from 1970s and was applied to medicine in the beginning, and is now routinely used for Non-Destruction Evaluation (NDE) [1]-[3]. Based on Huygens' principle [4]-[6], ultrasonic phased array offers a new method of scanning and inspection. Ultrasonic phased array takes the advantages over the traditional ultrasonic inspection that it is needless to replace probes regularly, and has wide scanning range and manipulates simply [7]-[10]. The material of the gas turbine blade is nickel base super alloy. Based on ultrasonic phased array, the samples made of nickel base super alloy were inspected and the detected images were analyzed to guide the inspection of gas turbine blades. Little attention has been devoted to the inspection method using ultrasonic phased array instrument and the image processing about the detected defects. Wang [11] noted that a region segmentation based method was presented to inspect the blades using radiographic testing (RT). However, his method is time-consuming and off-line detection, inconveniently. Meanwhile, the traditional approaches implementing ultrasonic phased array are normally lack of industry benchmarks and rely heavily on skilled technicians. The main parameters that describe the defects by the technicians in reference to analyzing defects are merely major axis, minor axis and categories of the defects, which have single indicator and relatively large error.

Considering this condition mentioned above, proposed in

Manuscript received April 9, 2016; revised August 5, 2016. The authors are grateful for the financial support provided by the National Basic Research Program of China (2013CB035704-3).

The authors are with State Key Laboratory for Manufacturing Systems Engineering, Xi'an, Shaanxi Province, China (e-mail: 463915175@qq.com, 20634698@qq.com,272282837@qq.com,1037693177@qq.com). this paper is a new method of analysis and processing data that the blade crown samples of the turbine blade were inspected to obtain the images using ultrasonic phased array instrument, and the accurate defects were finally recognized by filtering, edge detection and morphological operation etc. [12]-[15]. Then, quantitative and qualitative analysis and the location of the defects were discussed. The advantages of this approach are highlighted through comparing the results of this approach and the artificial evaluation.

\section{IMAGE ACQUIREMENT}

The ultrasonic phased array equipment shown in Fig. 1 is adopted, including an ultrasonic phased array device, an encoder, a probe, a wedge and a memorizer, whose parameters are shown in Table I.
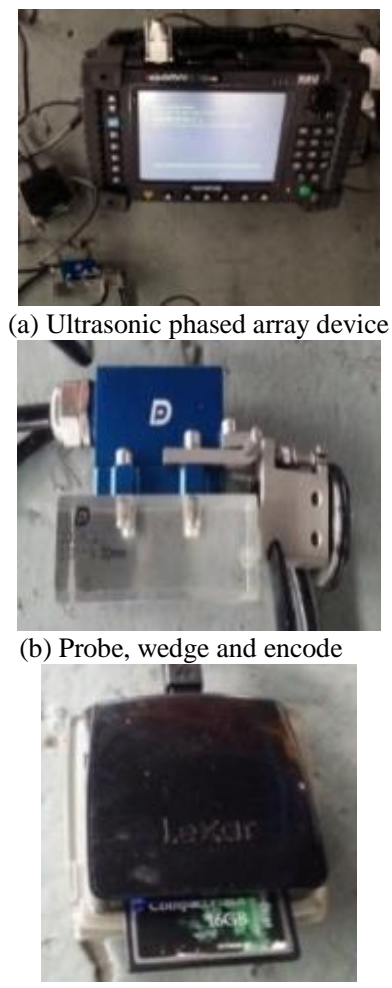

(c) Memorizer

Fig. 1. Ultrasonic phased array equipment.

The slices of the blade crown are acted as the experimental samples shown in Fig. 2 and their parameters are shown in Table II.

As shown in Fig. 3, the probe is applied from the top to the button when the samples are tested. The defect images obtained are showed in B-mode. The sections of the defects are displayed as the full lines in Fig. 3 and the detected images are displayed in Fig. 4. 
TABLE I:PARAMETERS OF UltRASONIC PHASEd ARRAY EQUIPMENT

\begin{tabular}{ccccccccccccc}
\hline Categories & Model & Resolution & $\begin{array}{c}\text { Steering } \\
\text { Angle }\end{array}$ & $\begin{array}{c}\text { Display } \\
\text { pattern }\end{array}$ & $\begin{array}{c}\text { Probe } \\
\text { type }\end{array}$ & Frequency & $\begin{array}{c}\text { Element } \\
\text { number }\end{array}$ & Shape & Thickness & Velocity & Material \\
\hline Parameters & $\begin{array}{c}\text { OmniScan } \\
\text { MX }\end{array}$ & $\begin{array}{c}264 \mathrm{um} * 264 \mathrm{u} \\
\mathrm{m}\end{array}$ & $0^{\circ}$ & B-mode & Linearity & $5 \mathrm{MHz}$ & 64 & Rectangle & $20 \mathrm{~mm}$ & $2337 \mathrm{~m} / \mathrm{s}$ & Polymer \\
\hline
\end{tabular}

TABLE II: PARAMETERS OF EXPERIMENTAL SAMPLES

\begin{tabular}{cccccc}
\hline Categories & Thickness & Material & Velocity & Near field & Focal depth \\
\hline Sample 1 & $10 \mathrm{~mm}$ & \multirow{2}{*}{ Nickel base super alloy } & $5700 \mathrm{~m} / \mathrm{s}$ & \multirow{2}{*}{$4.4 \mathrm{~mm}$} & $12 \mathrm{~mm}$ \\
Sample 2 & $12 \mathrm{~mm}$ & & & $12 \mathrm{~mm}$ \\
\hline
\end{tabular}

\section{IMAGE PREPROCESSING}

To analyze the gray level of the defects, the sample 1 is taken as an example. The gray level image shown in Fig. 5 is displayed by a dotted line through the defect. It can be seen from Fig. 4 and Fig. 5 that the detected images can be characterized as follows:

1) Blurring edges of defect and area;

2) Low contrast;

3) Containing scattering noise;

4) Changes in brightness consistent to changes in gray level.

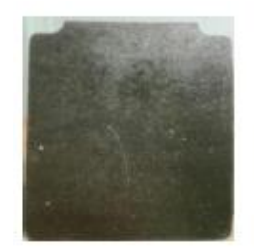

(a) Sample 1

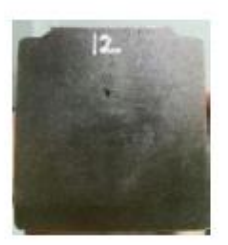

(b) Sample 2
Fig. 2. Experimental samples.

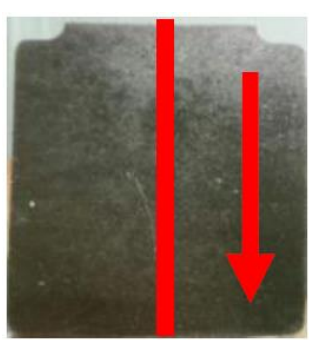

(a) Sample 1

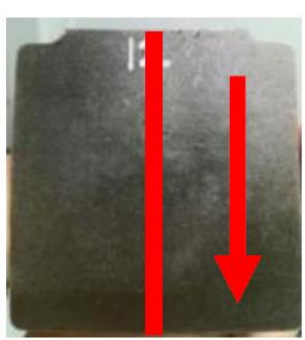

(b) Sample 2
: Scanning direction; : Scanning section Fig. 3. Probe scanning direction and display schematic diagram.

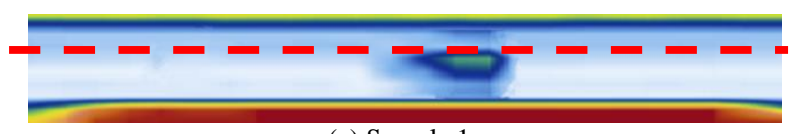

(a) Sample 1

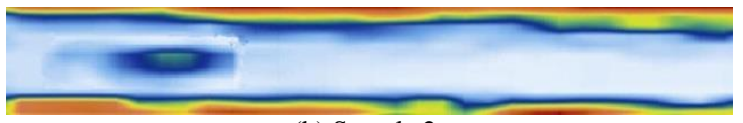

(b) Sample 2

- $:$ : A line through the defect

Fig. 4. Detected images.

Because of the reasons mentioned above, the original image should be preprocessed including homomorphic filtering and recognition of defects region. Homomorphic filtering aims to filter the scattering noise. Because of the initial wave and the primary reflection in the original image, the defect nearby region is loosely recognized to avoid the interference in the image processing.

Scattering is always inevitable in the cause of reflection and refraction because ultrasonic source is not an absolute point light source and the essence of ultrasonic inspection is the principle of reflection and refraction of ultrasound. It can lead to blurring image with low contrast. The scattering noise that is multiplicative noise is hardly filtered by spatial filtering, so homomorphic filtering is adopted in this paper. The principle of homomorphic filtering is shown in Fig. 6(a), the outcome of which is shown in Fig. 6(b).

Homomorphic filtering is an image processing method combining frequency filtering and gray alteration, depending on illuminance/reflectivity models as the basis of frequency processing and bettering the image quality by condensing brightness range and amplifying the contrast, which allows image processing to conform to the non-linear characteristic of the light response of human's eyes, avoiding the distortion of the image processed by Fourier transform directly.

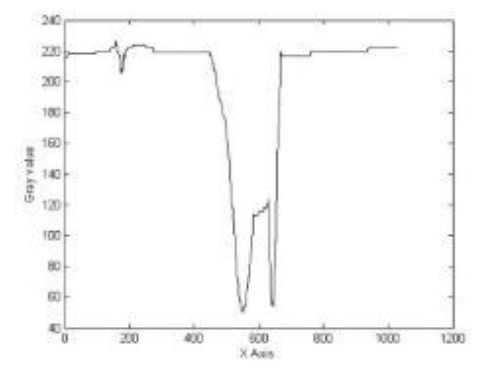

Fig. 5. The gray level through the defect.

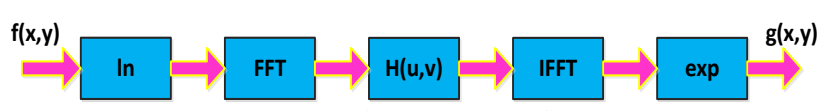

(a) Principle of homomorphic filtering

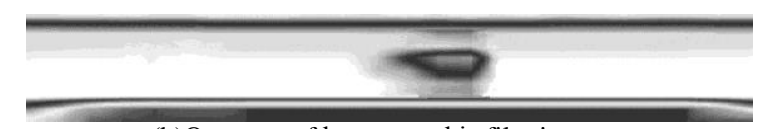

(b)Outcome of homomorphic filtering

Fig. 6. Homomorphic filtering.

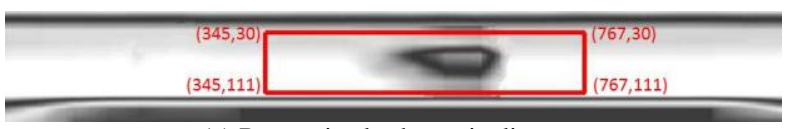

(a) Recognized schematic diagram

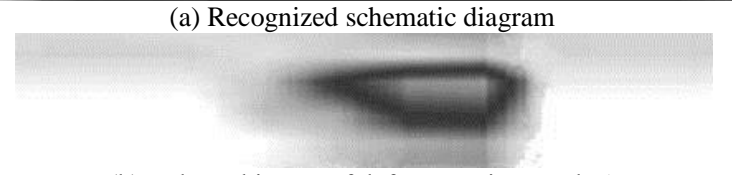

(b) Enlarged image of defect area in sample 1

Fig. 7. Recognition of defect region.

After filtering the mean gray level is decreased from 189.44 to 166.57 , while the standard deviation is decreased from 72.65 to 62.92 . Meanwhile, the peak signal-to-noise ratio is reduced by 21.14 , achieving excellent results.

To process and analyze image conveniently, the defect nearby region is loosely recognized from sample 1 due to the 
initial wave and primary reflection in the original image remained after homomorphic filtering, as Fig. 7(a) indicates the schematic diagram and Fig. 7(b) indicates the enlarged image of defect area. The defect will be recognized further in the next section.

\section{DEFECT RECOGNITION}

It can be seen from Fig. 7 that the defect region is obvious after homomorphic filtering and recognition. In this paper, the recognition of defects is implemented by four steps. Firstly, the defects are roughly recognized using Background Subtraction Method (BSM). Using Hough transform inspects whether the lines exist in the image. If exist, the false defects should be removed. Secondly, because of the broken outlines in the first step, the outlines of the defects should be fully connected and the interior outlines should be filled completely by morphology dilation and fill. Then, bilinear interpolation method is used to simulate the background of the defect in the original image. Finally, the background image subtracts the original image, obtaining the defects, and the accurate image is acquired.

\section{A. Background Subtract Method and Hough Transform}

Using the $15 \times 17$ average kernel, the background image shown in Fig. 8(a) is gained. The original image, then, subtracts the background image, obtaining the defects roughly, as shown in Fig. 8(b).

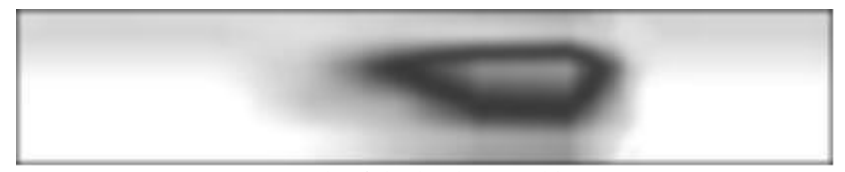

(a) Result of the background image

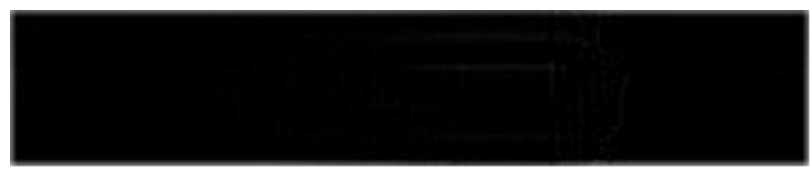

(b) Defects detected by BSM

Fig. 8. Background subtraction method.

It can be seen that the outlines of the defects detected by BSM cannot be seen clearly, so the differential operator is adopted to extract the outlines of the defect. This paper offers Canny operator to extract the outlines and the result is shown in Fig. 9.

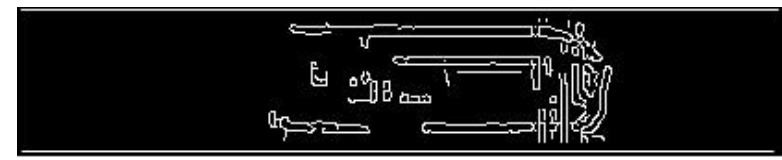

Fig. 9. Result of Canny operator.

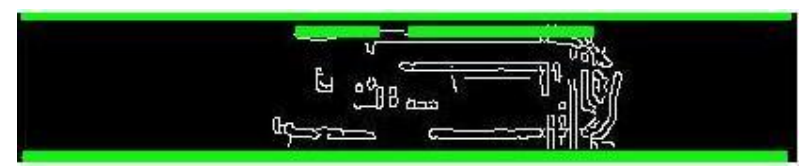

(a) Result of Hough transform

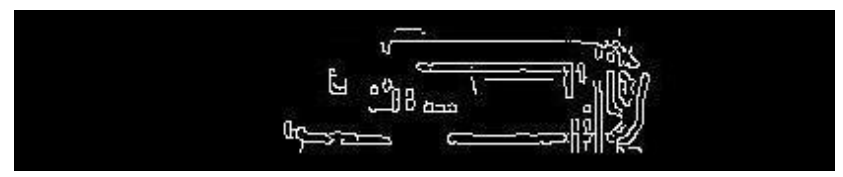

(b) Result that have removed the lines.

Fig. 10. Hough transform.
There are so many lines in the image that Hough transform should be used to detect the lines and remove them clearly. The result is shown in Fig. 10(a), the green parts of which are the lines detected, and the result that has removed the lines is shown in Fig. 10(b).

\section{B. Simulation Background}

Because of broken outlines from Fig. 10(b) the outlines of the defects are connected by morphology dilation and then the interior outline is fully filled by morphology fill to form a connective area shown in Fig. 11(a). The area in the original image; according to the same area in Fig. 10(b) are highlighted in black color as shown in Fig. 11(b). The background image shown in Fig. 11(c) is simulated by bilinear interpolation method whose principle is shown as follows:

$$
\mathrm{v}(x, y)=a x+b y+c x y+d
$$

where the four coefficients are determined by relative functions of the four adjoining points around the point $(x, y)$.

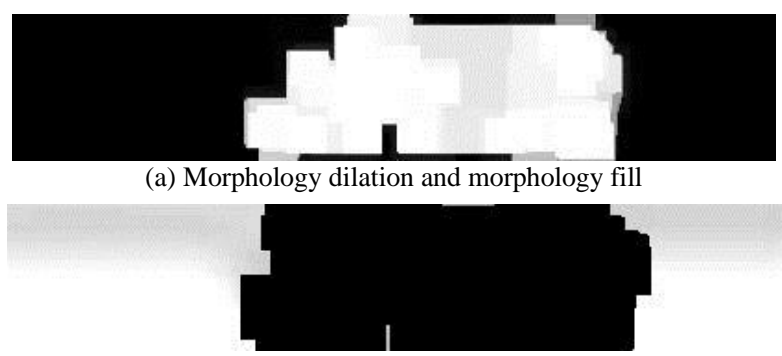

(b) Defect blacked in the original image

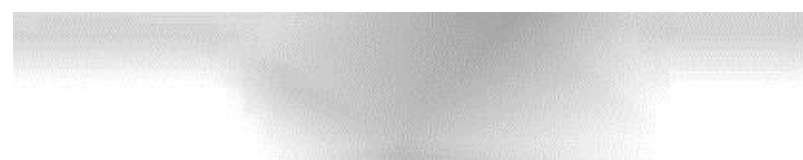

(c) The stimulated background

Fig. 11. Simulation background.

\section{Accurate Defects}

Here, the background image simulated subtracts the original image to recognize the defect image shown in Fig. 12(a). Based on the clustering theory, the Otsu's algorithm is used in this paper. After thresholding of the image as (2) by the obtained threshold, the defects can be detected accurately, as shown in Fig. 12(b). The principle of the Otsu's algorithm is shown as follows:

$$
\mathrm{g}(x, y)= \begin{cases}1, & f(x, y)>T \\ 0, & f(x, y)<T\end{cases}
$$

where $T$ is the best threshold.

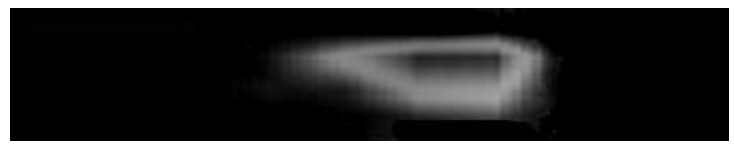

(a) Subtraction result

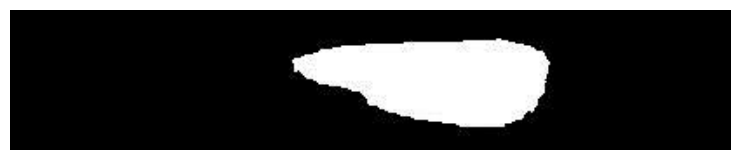

(b) Accurate defects

Fig. 12. Accurate defects

After the same procedure above, the defect shown in Fig. 13(a) of the sample 2 is recognized, and finally the accurate 
defect is shown in Fig. 13(b).

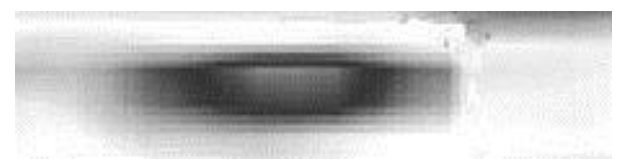

(a) The defect area of sample 2

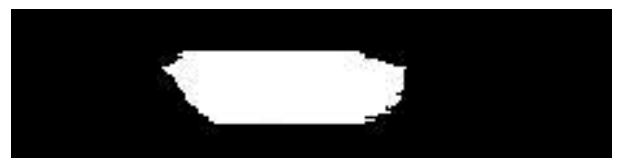

(b) The accurate defect of sample 2

Fig. 13. The original image and the accurate defect image of sample 2.

\section{DEFECT ANALYSES}

After the recognition of the defects, this section in this paper aims to analyze the defect including quantification, qualification and location analysis. The parameters of the quantitative analysis include area, perimeter, major axis and minor axis. The characteristic discrimination method and the quantitative discrimination method are used to determine the types of the defects in the qualitative analysis. And the main aim of the location analysis is to obtain the centroid coordinates of the defects.

\section{A. Quantitative Analysis}

The main geometric parameters that quantitatively describe the defects are area, perimeter, major axis and minor axis. The major axis is the maximum distance between the two pixels on the edge of the defect, while the minor axis is perpendicular to the major axis and has the longest distance of the two pixels on the edge of the defect. The four parameters can be obtained as formula (3) (6), respectively.

$$
\begin{aligned}
\mathrm{A} & =n_{a} \times a_{p} \\
P_{e} & =n_{e} \times s_{p} \\
L_{\text {max }} & =n_{\text {max }} \times s_{p} \\
L_{\text {min }} & =n_{\text {min }} \times s_{p}
\end{aligned}
$$

where: $\mathrm{A}$ - the area of the defect;

$P_{e}$ - the perimeter of the defect;

$L_{\max }$ - the major axis of the defect;

$L_{\min }$ - the minor axis of the defect;

$n_{a}$ - the number of pixels covered by defect;

$a_{p}$ - the area of each pixel;

$n_{e}$ - the number of pixels covered by the edge of defect;

$s_{p}$ - the size of the pixel.

The results of each parameter are shown in Table III.

TABLE III: QUANTITATIVE ANALYSIS RESULTS

\begin{tabular}{ccccc}
\hline Categories & Area $\left(\mathrm{mm}^{2}\right)$ & Perimeter(mm) & $\begin{array}{c}\text { Major } \\
\text { axis(mm) }\end{array}$ & $\begin{array}{c}\text { Minor } \\
\text { axis(mm) }\end{array}$ \\
\hline Sample 1 & 36.72 & 24.4 & 9.6 & 3.5 \\
Sample 2 & 27.42 & 16.7 & 6.2 & 4.1 \\
\hline
\end{tabular}

\section{B. Qualitative Analysis}

The two main defect types those are common in the interior blades are cracks and pores, which can be determined by two main methods, namely, characteristic discrimination method and quantitative discrimination method. The characteristic discrimination method is to judge the characteristics according to the cracks and pores. The pore is in the form of small holes, and the crack is a small and thin opening. Quantitative discrimination method uses the aspect ratio that represents the ratio of the major axis to the minor axis to determine the types of defects. If the aspect ratio is larger than or equal to 3 , the defect is a crack; if the aspect ratio is smaller than 3 , the defect is a pore. According to characteristic discrimination method, the accurate defects of the two samples are the small holes that conform to the characteristics of the pore, so they are both pores. According to quantitative discrimination method, the results are shown in Table IV.

TABLE IV: Results OF CHARACTERISTIC DisCRIMINATION METHOD

\begin{tabular}{ccccc}
\hline Categories & $\begin{array}{c}\text { Major } \\
\text { axis }(\mathrm{mm})\end{array}$ & $\begin{array}{c}\text { Minor } \\
\text { axis }(\mathrm{mm})\end{array}$ & $\begin{array}{c}\text { Aspect } \\
\text { ratio }\end{array}$ & Conclusion \\
\hline Sample 1 & 9.6 & 3.5 & 2.74 & pore \\
Sample 2 & 6.2 & 4.1 & 1.51 & pore \\
\hline
\end{tabular}

It is reasonable to see that the defect types of the two samples are both pores by synthesizing the two discrimination ways.

\section{Location Analysis}

In the case of the discrete image $f(x, y), p+q$ order moment $\left(m_{p q}\right)$ and central moment $\left(\mu_{\mathrm{pq}}\right)$ are defined as

$$
\begin{gathered}
m_{p q}=\sum_{j=1}^{N} \sum_{i=1}^{N} i^{p} j^{q} f(i, j) \\
\mu_{p+q}=\sum_{j=1}^{N} \sum_{i=1}^{N}\left(i-i_{c}\right)^{p}\left(j-j_{c}\right)^{q} f(i, j)
\end{gathered}
$$

where, the first order moment $\left(m_{10}, m_{01}\right)$ represents the centroid $\left(i_{c}, j_{c}\right)$ of the substance, so

$$
i_{c}=m_{10} / m_{00}, j_{c}=m_{01} / m_{00} .
$$

As for the region of the defects according to this principle mentioned above, the local centroid coordinates of the defects of the two samples are $(16.3 \mathrm{~mm}, 2.6 \mathrm{~mm})$ and $(11.3 \mathrm{~mm}, 9.9 \mathrm{~mm})$. The results are shown in Fig. 14 where the red stars represent the local centroid positions.

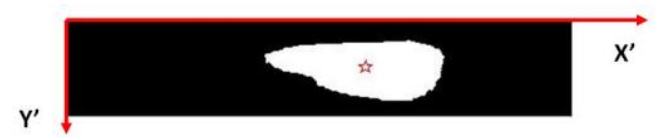

(a) Sample 1

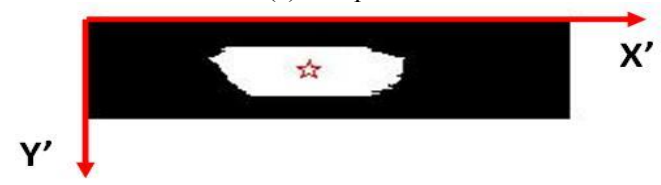

(b) Sample 2

Fig. 14. The centroid positions of the local coordinates system of the samples.

The centroid coordinates of the defects are $(39.6 \mathrm{~mm}$, $4.6 \mathrm{~mm})$ and $(19.3 \mathrm{~mm}, 5.3 \mathrm{~mm})$ after transforming the local coordinate system to the global coordinate system as shown 
in Fig. 15 where the red stars represent the centroid positions of the global coordinate system of the defects.

Finally, the two samples are viewed as regular hexahedrons and the centroid positions of the defects are represented in three dimensional forms, respectively, as shown in Fig. 16 where the red stars represent the centroid positions of the defects.

TABLE V: RESULTS oF THE DEFECT ANALYSIS

\begin{tabular}{|c|c|c|c|c|c|c|c|}
\hline Categories & Perimeter(mm) & $\operatorname{Area}\left(\mathrm{mm}^{2}\right)$ & $\begin{array}{c}\text { Major } \\
\operatorname{axis}(\mathrm{mm})\end{array}$ & $\begin{array}{c}\text { Minor } \\
\operatorname{axis}(\mathrm{mm})\end{array}$ & $\begin{array}{c}\mathrm{X} \\
\text { coordinate }(\mathrm{mm})\end{array}$ & $\begin{array}{c}\mathrm{Y} \\
\text { coordinate }(\mathrm{mm})\end{array}$ & Type \\
\hline Sample 1 & 24.4 & 36.72 & 9.6 & 3.5 & 39.6 & 4.6 & pore \\
\hline Sample 2 & 16.7 & 27.42 & 6.2 & 4.1 & 19.3 & 5.3 & pore \\
\hline Analysis & \multicolumn{4}{|c|}{ Quantitative analysis } & \multicolumn{2}{|c|}{ Location analysis } & Qualitative analysis \\
\hline
\end{tabular}

TABLE VI: RESULTS OF THE ARTIFICIAL EVALUATION

\begin{tabular}{|c|c|c|c|c|c|c|c|}
\hline Category & Perimeter(mm) & $\operatorname{Area}\left(\mathrm{mm}^{2}\right)$ & $\begin{array}{c}\text { Major } \\
\text { axis(mm) }\end{array}$ & $\begin{array}{c}\text { Minor } \\
\text { axis }(\mathrm{mm})\end{array}$ & $\begin{array}{c}\mathrm{X} \\
\text { coordinate }(\mathrm{mm})\end{array}$ & $\begin{array}{c}\mathrm{Y} \\
\text { coordinate }(\mathrm{mm})\end{array}$ & Type \\
\hline Sample 1 & - & - & 9.6 & 3.5 & - & - & pore \\
\hline Sample 2 & - & - & 6.2 & 4.1 & - & - & pore \\
\hline Analysis & \multicolumn{4}{|c|}{ Quantitative analysis } & \multicolumn{2}{|c|}{ Location analysis } & Qualitative analysis \\
\hline
\end{tabular}

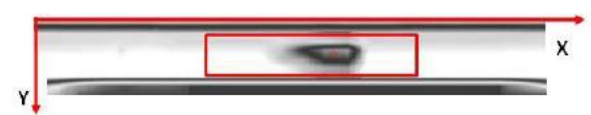

(a) Sample 1

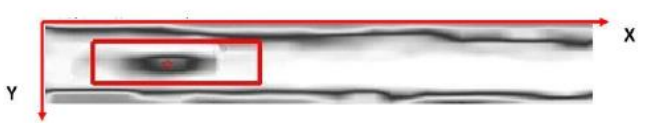

(b) Sample 2

Fig. 15. The centroid positions of the global coordinates system of the samples.

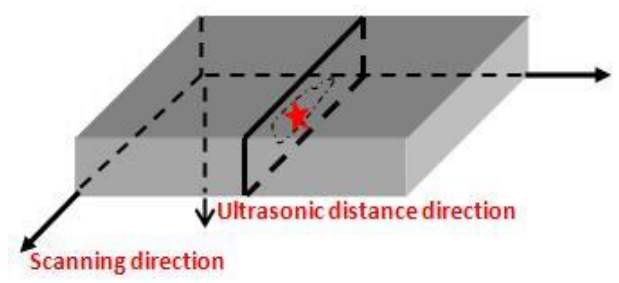

(a) Sample 1

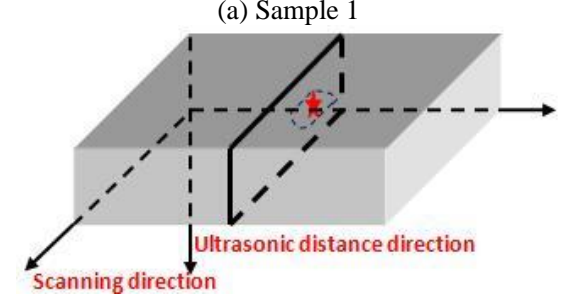

(b) Sample 2

Fig. 16. The centroid positions of the two samples in three dimensional forms.

\section{RESUlts ANALYSIS}

The analysis results of the defects are summarized in Table $\mathrm{V}$. And the results of the artificial evaluation are shown in Table VI.

By comparing the two kinds of the results, the artificial evaluation is able to roughly estimate the major axis and the minor axis, unable to estimate the area, the perimeter and the centroid coordinates of the defects. The types of the defects can be merely estimated by characteristic discrimination method, which is a single approach and is easy for erroneous judgement. Advantages of this approach proposed in this paper are that it not only offers the parameters like area, perimeter, major axis and minor axis, but also analyzes the centroid coordinates and the positions of the defects, even offers the types of the defects finally by combining the two discrimination methods, avoiding the erroneous judgement. As a result, with various parameters, high accuracy and reliability, the method in this paper is an excellent one.

\section{CONCLUSIONS}

In this paper the samples made of nickel base super alloy are inspected by ultrasonic phased array. The accurate defects are finally obtained and analyzed by quantification, qualification and location analysis. Compared with the results of the artificial evaluation, the method in this paper offers more parameters including perimeter, area, major axis and minor axis and the location in the samples and the types of the defects. However, the artificial evaluation only offers major axis and minor axis and the types of the defects which are evaluated by characteristic method and need more standards. To conclude, this method in this paper is a good method with a good many of parameters and reliability.

\section{REFERENCES}

[1] P. Crowther, "Practical experience of phased array technology for power station applications," Insight, vol. 9, pp. 525-528, 2004.

[2] B. W. Drinkwater and P. D. Wilcox. "Ultrasonic arrays for non-destructive evaluation: A review," NDT \& E International, vol. 7, pp. 525-541, 2006

[3] Q. S. Yuan, Y. M. Guo, Z. G. Sun et al., "Development of an ultrasonic phased array for nondestructive testing of pipes: Theory and practice," Materials Evaluation, vol. 4, pp. 501-506, 2011.

[4] A. Fenster, G. Parraga, and J. Bax, "Three-dimensional ultrasound scanning," Interface Focus, vol. 4, pp. 503-519, 2011.

[5] X. E. Gros, N. B. Cameron, and M. King, "Current applications and future trends in phased array technology," Insight, vol. 11, pp. 673-678, 2002.

[6] C. Holmes, B. W. Drinkwater, and P. D. Wilcox, "Post-processing of the full matrix of ultrasonic transmit-receive array data for non-destructive evaluation," Ndt \& E International, vol. 8, pp. 701-711, 2005 . 
[7] C. Holmes, B. W. Drinkwater et al., "Advanced post-processing for scanned ultrasonic arrays: Application to defect detection and classification in non-destructive evaluation," Ultrasonics, vol. 6-7, pp. 636-642, 2008.

[8] R. Huang and L. W. Schmerr. "Characterization of the system functions of ultrasonic linear phased array inspection systems," Ultrasonics, vol. 2, pp. 219-225, 2009.

[9] S. J. Jin et al., "Development and application of ultrasonic phased array inspection technology," Journal of Electronic Measurement and Instrument, vol. 9, pp. 925-934, 2014.

[10] M. Moles and A. Lamarre, "Ultrasonic phased arrays," Advanced Materials \& Processes, vol. 3, pp. 37-40, 2007.

[11] Y. G. Wang et al., "Region segmentation based radiographic detection of defects for gas turbine blades," presented at 2015 IEEE International Conference on Mechatronics and Automation, 2015.

[12] D. Li, L. Q. Liang, and W. J. Zhang, "Defect inspection and extraction of the mobile phone cover glass based on the principal components analysis," International Journal of Advanced Manufacturing Technology, vol. 9-12, pp. 1605-1614, 2014.

[13] J. Y. Liu, Q. J. Tang, Y. Wang et al., "Defects' geometric feature recognition based on infrared image edge detection," Infrared Physics \& Technology, vol. 67, pp. 387-390, 2014.

[14] R. Saxena et al., "Algorithm and technique on various edge detection: A survey," Signal \& Image Processing, vol. 3, pp. 65-75, 2013.

[15] S. Uchida, "Image processing and recognition for biological images," Development Growth \& Differentiation, vol. 4, pp. 523-549, 2013.

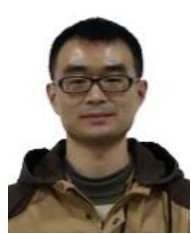

Mengqiu Gao was born in Suqian, Jiangsu Province, on Nov. 16, 1990. He received the bachelor degree in mechanical engineering in June, 2014 from Zhengzhou University in Zhengzhou, Henan Province.

$\mathrm{He}$ now studies in Xi'an Jiaotong University as a postgraduate student. His research interests include NDT and ultrasonic phrase.

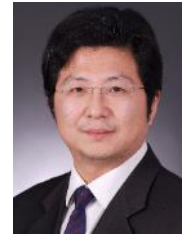

Bing Li was born in Xi'an, Shannxi Province, in Oct 1965. He received the Ph.D degree in mechanical engineering in Dec. 2004 from Xi'an Jiaotong University in Xi'an, Shannxi Province.

He now is a professor in Xi' an Jiaotong University. His research interests include NDT and precision measurement technology.

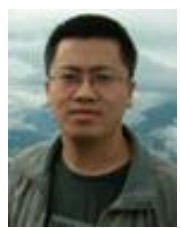

Lei Chen was born in Harbin, Heilongiiang Province, in May 1980. He received the Ph.D degree in mechanical engineering in June 2014 from Xi'an Jiaotong University in Xi'an, Shannxi Province.

He now is a lecturer in Xi'an Jiaotong University. His research interests include NDT and precision measurement technology.

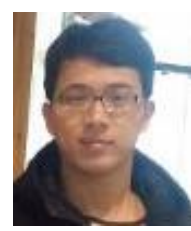

Yuegen Wang was born in Taiyuan, Shanxi Province, in Jan. 1989. He received the bachelor degree in mechanical engineering in June 2013 from Xi'an Jiaotong University in Xi'an, Shannxi Province.

He now studies in Xi'an Jiaotong University as a postgraduate student. His research interests include NDT and X-ray Inspection. 